\title{
Availability of Sugar Solutions for Colony Development and Progeny- Queen Production of the European Bumblebee, Bombus terrestris
}

\author{
Hyung Joo Yoon*, Kyeong Yong Lee, Mi Ae Kim, Sang Mi Han, Mi Young Ahn, and In Gyun Park \\ Applied Entomology Division, Department of Agricultural Biology, National Academy of Agricultural Science, Suwon 441- \\ 100, Korea
}

(Received 18 February 2012; Accepted 10 March 2012)

Bumblebees are widely used to pollinate various crops, especially tomato, in greenhouses and fields. Here, we investigated whether different sugar solutions have any effects on the oviposition and colony development of Bombus terrestris queens. The types of sugar solution used were a white sugar solution, brown sugar solution, dark brown sugar solution, and prepared sugar solution; all solution contained with a $\mathbf{4 0 \%}$ sugar concentration and $0.3 \%$ sorbic acid. Among these sugar solutions, $B$. terrestris queens reared on the white sugar solution exhibited the best results; the rates of oviposition, colony foundation and progeny-queen production were 75.0 $\pm 11.0,33.0 \pm 9.6$ and $21.7 \pm 7.3$, respectively, which corresponded to 1.1-33.0-fold increases over the results of other sugar solutions. Queens reared on the prepared sugar solution did not found any colonies. The death rate within one month was lowest for the queens reared on the white sugar solution at $26.1 \pm 10.9 \%$, which was 1.2-2.0-fold lower than the rate for the other sugar solutions. Furthermore, the numbers of progeny produced by queens reared on the white sugar solution, $131.4 \pm 38.8$ workers and $51.0 \pm 40.6$ queens, were also higher, corresponding to 1.1-1.2-fold increases compared to queens raised on the other sugar solutions. Therefore, the white sugar solution was the favorable sugar solution for the egg-laying and colony developmental characteristics of $B$. terrestris queens.

Key words: Bumblebee, Bombus terrestris, Sugar solution, Oviposition, Colony development, Progeny-queen production.

\footnotetext{
*To whom the correspondence addressed Department of Agricultural Biology, National Academy of Agricultural Science, RDA, Suwon 441-100, Korea. Tel: +82-31-290-8567; E-mail: yoonhj1023@korea.kr
}

\section{Introduction}

Bumblebees (Hymenoptera, Apidae, Bombini) play a major ecological role as important pollinators of various greenhouse crops and are particularly effective at pollinating tomatoes (Buchmann and Hurley, 1978; Banda and Paxton, 1991; Free, 1993). In general, Bombus species are annual eusocial insects with short-lived colonies and are found mainly in Palaearctic, Nearctic, and tropical ecosystems. Queens are the only caste to overwinter (enter diapause), and workers and males die during late summer and early autumn, respectively (Heinrich, 1979, Duchateau and Velthusis, 1988; Goulson, 2003). Unlike in honeybees and stingless bees, bumblebee colonies are generally founded by a single queen and thus pass through stages in which a relatively small number of individuals must meet colony needs (Michener, 1974). Foraging regulation is a prime example of colony cooperative action. Bumblebee colonies can activate nectar foraging and adjust the number of nectar foragers according to the colony's nectar stores. Foraging activity increases in Bombus terrestris and $B$. transversalis colonies after foragers return from nectar collection (Dornhaus and Cameron, 2003), and this increase depends on the food sucrose concentration (Dornhaus and Chittka , 2005).

In studies of the artificial mass-rearing of bumblebees, every author has used pollen and honey obtained from honeybee hives (Griffin et al., 1991; Tasei and Aupinel, 1994; Ono et al., 1994; Hannan et al., 1998, Yoon et al., 2005a, b) although the supplying method has varied slightly among experiments. Pollen and nectar are the two food resources necessary for bumblebees. Pollen is their primary protein source and is necessary for larval growth and the production of eggs by reproductive females, whereas nectar is their major source of energy for activity and the thermoregulation of the hive (Plowright and Pendrel, 1977; Heinrich, 1979; Sutcliff and Plowright, 1988, 
1990). Both resources are initially acquired by the queen during colony development and later by foraging workers. The study of feeding is significant for efforts to enhance colony development. In B. ignitus, to deposit food on larvae, workers place their mouths into the larval cell through an orifice (Katayama, 1973, 1975). In the majority of cases, the number of orifices in the wax envelope made by the queen and/or workers when feeding the larvae corresponded to the number of larvae present in the egg cell. Queen-worker differentiation in social insects depends primarily on nutritional and social factors (De Wilde and Beetsma, 1982; Wheeler, 1986). The production of new bumblebee queens has been related to factors such as the availability of food, the relative number of workers involved in foraging and nursing and the presence of a queen (Pomeroy and Plowright, 1982; Duchateau and Velthuis, 1988). Caste-specific differences in feeding frequencies are a result, but not the cause, of differences in development (Pereboom et al., 2003). As the survival, growth and reproduction of a colony depend strongly upon the influx of nectar and pollen, selection is expected to maximize energy influx into the colony by efficient allocation of the available workforce to the necessary tasks (Spaethe and Weidenmüller, 2002). Short-term food shortfalls affect colony development in many ways. They may increase the vulnerability of colonies to predators and parasites (Cartar and Dill, 1991), provoke a drop in brood temperature and torpor in workers (Heinrich, 1979), lengthen the development time of immatures (Plowright and Pendrel, 1977; Sutcliffe and Plowright, 1988, 1990), and result in the production of smaller adults (Sutcliffe and Plowright, 1990). Workers in nectar-dependent bumblebee colonies may switch from in-colony to foraging tasks (Cartar, 1992), take more risks by foraging in the presence of predators (Cartar, 1991), and be less aggressive towards predators and social parasites (Cartar and Dill, 1991).

In a previous paper (Yoon et al., 2005b), we discussed different concentrations of sugar solution and the addition of antiseptic to the sugar solution. Therefore, in this study, we investigated whether different types of sugar solutions have any effects on oviposition and colony development in $B$. terrestris.

\section{Materials and Methods}

\section{Origin of experimental insects}

The experimental insects were $13^{\text {th }}$ generation $B$. terrestris queens that had been either $\mathrm{CO}_{2}$ treated or artificially hibernated for 10 weeks. The $\mathrm{CO}_{2}$-narcosis group was exposed to $99 \% \mathrm{CO}_{2}$ for 30 min daily for two consecutive days (Yoon et al., 2003). The artificially hibernated queens were hibernated for 10 weeks at $2.5^{\circ} \mathrm{C}$ while preserved in a bottle filled with perlite and kept at approximately $80 \%$ R.H. Next, the queens were placed in flight cages for three days and then reared at $27 \pm 1^{\circ} \mathrm{C}$ and $65 \%$ R.H.

\section{Indoor rearing}

The basic colony-rearing technique followed that described in Yoon et al. (2002). The queens were reared in three types of plastic boxes (1.5 mm thick), one each for nest initiation $(10.5 \times 14.5 \times 6.5 \mathrm{~cm})$, colony foundation $(21.0$ $\times 21.0 \times 15.0 \mathrm{~cm})$, and colony maturation $(24.0 \times 27.0 \times$ $18.0 \mathrm{~cm}$ ). Queens were first confined individually in small boxes for colony initiation and remained there until oviposition. To stimulate egg-laying, two old and narcotized B. terrestris workers, aged 10-20 days after emergence, were added to each box with a queen (Yoon and Kim, 2002). When the adults from the first brood emerged, the nest was transferred to a medium box for colony foundation and left there until the number of workers reached 50. The nest was then moved to a larger box for further colony development. A forty percent sugar solution with $0.2 \%$ sorbic acid and pollen dough were provided ad libitum (Yoon et al., 2005a). The pollen dough was made from a sugar solution and pollen $(\mathrm{v}: \mathrm{v}=1: 1)$.

\section{Egg-laying characteristics and colony development in $B$. terrestris under different sugar solutions}

To examine the effects of the sugar solution on egg-laying characteristics of $B$. terrestris, the following environmental conditions were provided. The sugar solution regimes were a white sugar solution, brown sugar solution, dark brown sugar solution, and prepared sugar solution (prepared with dextrose, fructose, sucrose, etc.). The concentrations of the sugar solutions were $40 \%$, based on the results of a previous paper (Yoon et al., 2005a). The antiseptic was sorbic acid (Junsei Chemical Co.), which is used in artificial diets of insects, and its concentration was $0.2 \%$. The experimental insects were $13^{\text {th }}$ generation $B$ terrestris queens that had been either $\mathrm{CO}_{2}$-treated or artificially hibernated for 10 weeks. In the case of the $\mathrm{CO}_{2}$-treated queens, on the second day after mating, queens were $\mathrm{CO}_{2}$-treated and then reared in a climate-controlled room $\left(27 \pm 1^{\circ} \mathrm{C}, 65 \%\right.$ R.H. and continuous darkness). A total of 30 queens were used in that experiment with two replications. On the other hand, artificially hibernated queens were placed in flight cages for three days (Yoon et al., 2004b) and then reared in a climatecontrolled room $\left(27 \pm 1^{\circ} \mathrm{C}, 65 \%\right.$ R.H. and continuous darkness). A total of $40 \mathrm{~B}$. terrestris queens were used in the artificial hibernation experiment. There were no significant differences in the weight of the queens before rearing them on different sugar solutions (0.74-0.76 g) at $p<0.05$ using Tukey's pairwise comparison test (Fig. $1, \mathrm{~F}=0.46, \mathrm{df}=3$, 


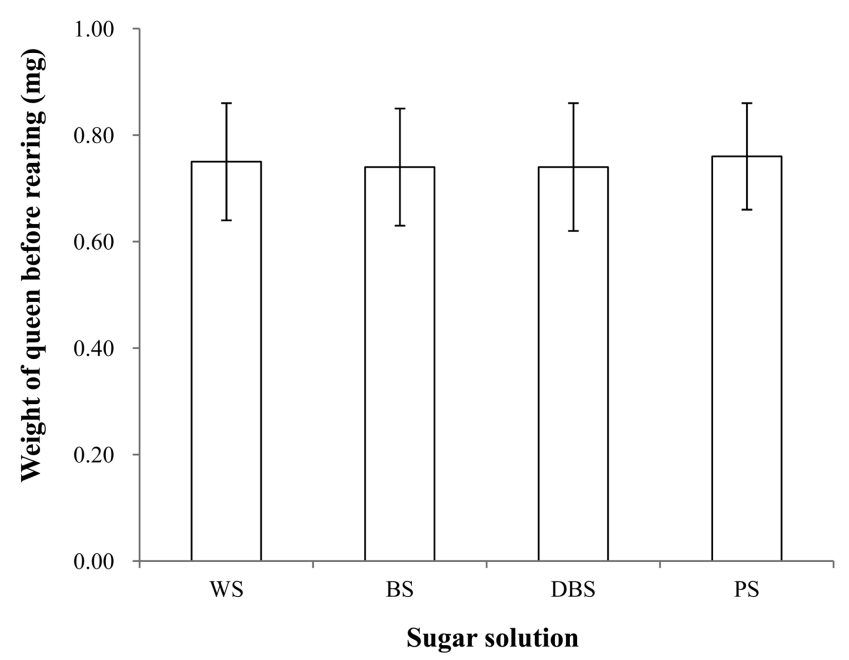

Fig. 1. Body weights of $B$. terrestris queens before rearing them on different sugar solutions. Abbreviations: WS, white sugar solution; BS, brown sugar solution; DBS, dark brown sugar solution, PS, prepared sugar solution. There were no significant differences in the weights of queen before rearing them on different sugar solutions based on a significance level of $p<0.05$ using Tukey's pairwise comparison test. The number of B. terrestris queens allotted to this experiment was 30 and there were 3 replicates.

$396, p=0.707)$. The egg-laying characteristics of each colony were estimated by the rate of oviposition and the preoviposition period. The queens that did not oviposit in 40 days were excluded from the number of oviposited colonies (Yoon et al., 2004a). The developmental ability of each colony was estimated based on the rates of colony foundation and progeny-queen production, the period of first adult emergence and the number of adults produced. Here, colony foundation was defined as the time period when more than 50 workers emerged from a colony.

\section{Statistical analyses}

Statistical analyses were conducted using Tukey's pairwise comparison tests following one-way ANOVA (MINITAB Release 13 for Windows, 2000). Tukey's pairwise comparison test was used to examine colony development in B. terrestris with reference to the effects of sugar solutions, the time spans until colony foundation and first adult emergence, and the number of adults produced.

\section{Results and Discussion}

Egg-laying characteristics of $B$. terrestris queens raised on different sugar solutions

We investigated the effect of sugar solution on the ovi-

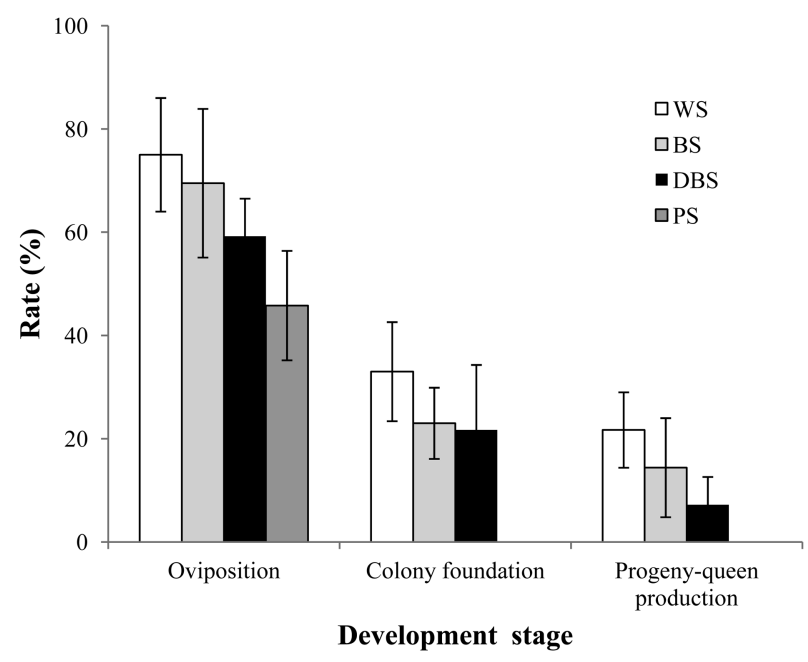

Fig. 2. The colony development of B. terrestris queens indoorreared on different sugar solutions. For abbreviations, see the legend to Fig. 1. There were no significant differences in rates of oviposition, colony foundation or progeny-queen production among different sugar solutions at $p<0.05$ using a Chi-square test. The number of $B$. terrestris queens allotted to this experiment was 30, and there were 3 replicates.

position rate and preoviposition period of $B$. terrestris queens (Fig. 2, Table 1). Among the four sugar solutions, the oviposition rate of queens reared on the white sugar solution was the highest at $75.0 \pm 11.0 \%$, followed by the brown sugar solution, dark brown sugar solution and prepared sugar solution. This value corresponded to 1.1-1.6fold increases over the results of the other sugar solutions, although there was no statistically significant difference in the oviposition rates among the different sugar solutions (Tukey's pairwise comparison test: $\mathrm{F}=4.00, \mathrm{df}=3,8, p$ $=0.052$ ) (Fig. 2). The preoviposition period of the queens reared on brown sugar solution was $16.1 \pm 9.5$ days, which was 3.1-6.2 days shorter than that of queens reared on the other sugar solutions (Table 1). The preoviposition period of $B$. terrestris queens was significantly affected by the type of sugar solution (Tukey's pairwise comparison test, $\mathrm{F}=5.48, \mathrm{df}=3,263, p=0.001)$. Regarding the death rate within one month (Fig. 3), the rate for queens reared on the white sugar solution was the lowest at $26.1 \pm$ $10.9 \%$, which was $1.2-2.0$-fold lower than the rates associated with the sugar solutions. This difference in the death rate within one month was statistically significant $(\mathrm{F}$ $=4.92, \mathrm{df}=3,8, p=0.032$ ).

In view of the above results, the queens raised on the white sugar solution had better egg-laying characteristics than did the queens reared on other sugar solutions. Although egg-laying is only one small step in the process 
Table 1. Lengths of periods of preoviposition and colony foundation for B. terrestris queens indoor-reared on different sugar solutions

\begin{tabular}{ccccc}
\hline Sugar solution & $\mathrm{n}$ & Preoviposition (days) & $\mathrm{n}$ & Colony foundation (days) \\
\hline WS & 93 & $14.9 \pm 8.2 \mathrm{a}$ & 27 & $48.6 \pm 5.5$ \\
\hline BS & 68 & $11.8 \pm 6.9 \mathrm{a}$ & 27 & $52.3 \pm 7.4$ \\
\hline DBS & 60 & $16.1 \pm 9.5 \mathrm{ab}$ & 22 & $51.8 \pm 6.6$ \\
\hline PS & 47 & $18.0 \pm 9.6 \mathrm{ab}$ & - & - \\
\hline
\end{tabular}

1) $n=$ number of colonies surveyed.

2) For abbreviations, see the legend for Fig. 1.

3) The differences in the preoviposition periods of $B$. terrestris queens raised on different sugar solutions were significant at $p>$ 0.05 using Tukey's pairwise comparison test.

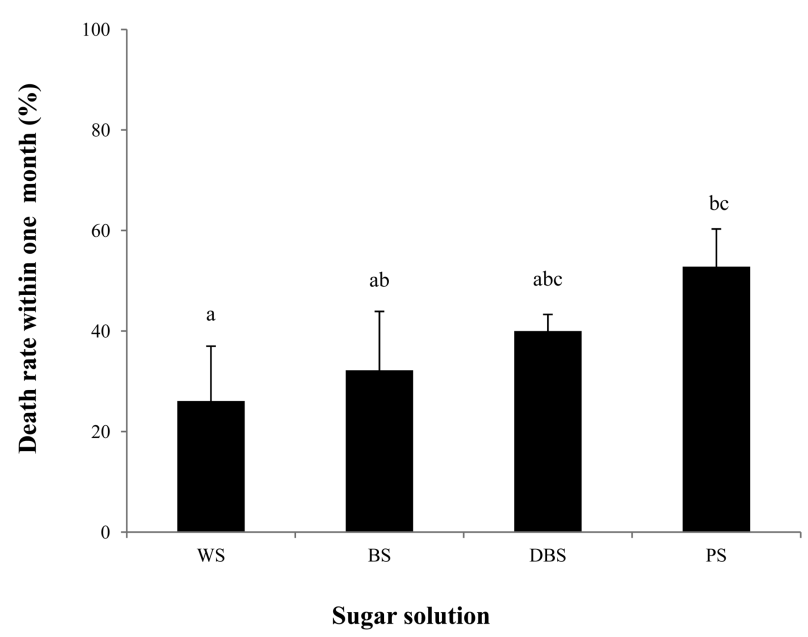

Fig. 3. Death rate within one month of $B$. terrestris queens indoor-reared on different types of sugar solutions. For abbreviations, see the legend to Fig. 1. The differences in death rate within one month between different sugar solutions were significant at $p>0.05$ using Tukey's pairwise comparison test.

of successfully rearing a bumblebee, it is a key factor in the large-scale production of commercial colonies and can reduce production costs. Yoon et al. (2004a) reported that a queen having an early first oviposition day could make a colony stronger and could make the colony formation period shorter. Additionally, they suggested that the first oviposition day is an important factor in the successful artificial rearing of bumblebees.

\section{Colony development of $B$. terrestris queens raised on different sugar solutions}

The colony development of $B$. terrestris queens raised on different sugar solutions was also investigated (Fig. 2, Table 1). In terms of the rate of colony foundation, which is one of the main criteria for colony quality in commercial rearing, the white sugar solution queens exhibited the best performance, $33.0 \pm 9.6 \%$, which was $1.4-33.0$-fold higher than the rates of queens raised on the other sugar solutions and was followed by the brown sugar solution, dark brown sugar solution and prepared sugar solution at $23.0 \pm 6.9 \%, 21.7 \pm 12.6 \%$ and $0 \%$, respectively. Queens reared on the prepared sugar solution did not found any colonies. There was a statistically significant difference in the colony foundation rates of $B$. terrestris queens raised on the different sugar solutions $(\mathrm{F}=5.59, \mathrm{df}=3,8, p=$ 0.0.23). As illustrated in Fig. 2, the rate of progeny-queen production of the white sugar solution queens was $21.7 \pm$ $7.3 \%$; this value was $1.5-21.7$-fold higher than the rates for the brown sugar solution, dark brown sugar solution and preparation sugar solution queens. The progenyqueen production rate of $B$. terrestris queens was significantly affected by the type of sugar solution provided ( $\mathrm{F}$ $=5.97, \mathrm{df}=3,8, p=0.019)$. The colony foundation period of queens reared on the brown sugar solution was $48.6 \pm$ 5.5 days, which was 3.2-3.8 days shorter than the periods of queens reared on the other sugar solutions (Table 1). The colony foundation period of $B$. terrestris queens was significantly affected by the type of sugar solution provided $(\mathrm{F}=1.70$, $\mathrm{df}=2,263, p=0.191)$. Table 2 summarizes the time of first adult emergence for $B$. terrestris utilizing different sugar solutions. The period before the emergence of the first worker was the shortest for the prepared sugar solution queens among all sugar solutions $(23.8 \pm 2.1$ days $)$, followed by the dark brown sugar solution, brown sugar solution and white sugar solution. There was a statistically significant difference detected for the period before first worker emergence between sugar solutions $(\mathrm{F}=23.51, \mathrm{df}=3,51, p=0.0001)$. The period before the first emergence of a male, which ranged from 46.8-56.3 days, was not significantly different between sugar solutions $(\mathrm{F}=1.62, \mathrm{df}=2,79, p=0.204)$. Similarly, the period before the first emergence of a queen, which was 65.9-70.7 days, was also not affected by the type of sugar solution $(\mathrm{F}=0.53$, df $=2,44, p=0.592)$.

The relationship between the number of adults produced and sugar solutions was investigated by comparing the 
Table 2. Time until the emergence of the first adult for B. terrestris queens indoor-reared on different sugar solutions

\begin{tabular}{ccccccc}
\hline \multirow{2}{*}{$\begin{array}{c}\text { Sugar } \\
\text { solution }\end{array}$} & $\mathrm{n}$ & Worker & $\mathrm{n}$ & Male & $\mathrm{n}$ & Queen \\
\cline { 2 - 7 } & 54 & $25.8 \pm 4.3 \mathrm{ab}$ & 38 & $56.3 \pm 20.7$ & 25 & $69.7 \pm 13.3$ \\
\hline WS & 37 & $25.2 \pm 4.3 \mathrm{ac}$ & 30 & $49.7 \pm 16.9$ & 14 & $70.7 \pm 10.1$ \\
\hline BS & 33 & $25.7 \pm 5.9 \mathrm{bcd}$ & 14 & $46.8 \pm 20.7$ & 7 & $65.9 \pm 7.9$ \\
\hline DBS & 4 & $23.8 \pm 2.1 \mathrm{~d}$ & - & - & - & - \\
\hline PS & &
\end{tabular}

1) $n=$ number of colonies surveyed.

2) For abbreviations, see the legend for Fig. 1.

3) The differences in the time of emergence of the first adult for B. terrestris queens raised on different sugar solutions were significant at $p>0.05$ using Tukey's pairwise comparison test."

Table 3. Number of adults produced and longevity of foundation for B. terrestris queens indoor-reared on different sugar solutions

\begin{tabular}{|c|c|c|c|c|c|c|}
\hline \multirow{2}{*}{$\begin{array}{l}\text { Sugar } \\
\text { solution }\end{array}$} & \multicolumn{4}{|c|}{ Number of adults produced } & \multirow{2}{*}{$\mathrm{n}$} & \multirow{2}{*}{$\begin{array}{c}\text { Longevity of } \\
\text { foundation queen (days) }\end{array}$} \\
\hline & $\mathrm{n}$ & Worker & $\mathrm{n}$ & Queen & & \\
\hline WS & 24 & $131.4 \pm 38.8$ & 22 & $51.0 \pm 40.6$ & 57 & $80.0 \pm 20.5 \mathrm{a}$ \\
\hline $\mathrm{BS}$ & 27 & $120.5 \pm 43.7$ & 15 & $48.2 \pm 36.8$ & 34 & $81.7 \pm 18.5 \mathrm{a}$ \\
\hline DBS & 11 & $114.3 \pm 38.2$ & 7 & $43.3 \pm 15.7$ & 39 & $88.1 \pm 15.8 \mathrm{a}$ \\
\hline PS & - & - & - & - & 6 & $53.8 \pm 8.8 b$ \\
\hline
\end{tabular}

1) $n=$ number of colonies surveyed.

2) For abbreviations, see the legend for Fig. 1.

3) The differences in the longevity of foundation of queens raised on different sugar solutions were significant at $p>0.05$ using Tukey's pairwise comparison test.

time it took for more than 50 workers to emerge from each colony (Table 3). The numbers of workers reared on the white sugar solution was $131.4 \pm 38.8$, which was 1.1 -fold that of the other wake-up treatments. The prepared sugar solution queens did not produce workers or queens. There was no statistically significant difference between the sugar solutions $(\mathrm{F}=0.80, \mathrm{df}=2,24, p=0.454)$. The production of workers is important in evaluating the potential pollination efficiency of an insect pollinator. Regarding the number of queens produced, which is an important factor in the year-round rearing of bumblebees, the white sugar solution queens produced $51.0 \pm 40.6$; this corresponded to an 1.1-1.2-fold increase over queens raised on the other sugar solutions. The longevity of the foundation of queens reared on the brown sugar solution was $53.8 \pm$ 8.8 days, which was 26.2-34.3 days shorter than those of the queens reared on the other sugar solutions, and was followed by the white sugar solution, brown sugar solution and dark brown sugar solution at $80.0 \pm 20.5 \%, 81.7$ $\pm 18.5 \%$ and $88.1 \pm 15.8 \%$, respectively (Table 3 ). The size of a bumblebee worker affects her physiological abilities, including thermoregulation (Bishop and Armbruster, 1999; Heinrich and Heinrich, 1983), flight speed (Pyke, 1978) and nectar ingestion rate (Harder, 1983). The size of a worker is important to a colony because large workers are needed to perform efficiently as foragers; only large insects can regulate their body temperature effectively for foraging in inclement weather, whereas small workers tend to stay inside the nest and attend to nest duties (Heinrich, 1979; Sutcliffe and Plowright, 1988; Spaethe and Weidenmüller, 2002). Small workers usually forage only when the population of larger foragers has been depleted (Brian, 1952). Larger bees may reach the nectaries more easily and extract the nectar faster because of their longer tongues and stronger sucking-related muscles (Winston, 1979; Harder, 1983). Queens grow larger than other bees because they obtain more food than worker larvae. This can partly be explained by the longer duration of their development and, directly related to this, the higher frequency of feeding during the last instar (Röseler and Röseler, 1974; Ribeiro et al., 1999). Ono et al. (1994) demonstrated that sucrose was converted into fructose and glucose within a few minutes by adding macerated hypopharyngeal glands from $B$. terrestris workers. In the case of bumblebees, sucrose concentration takes precedence over warmth, so that when there is a difference in sucrose concentration between food sources, bees will typically choose the sweeter feeder, even if the less sweet 
feeder is several degrees warmer (Whitney et al., 2008). The longevity of foundation of $B$. terrestris queens was significantly affected by sugar solutions $(\mathrm{F}=4.23, \mathrm{df}=3$, $132, p=0.007$ ).

In view of the above results related to colony developmental characteristics, the most favorable sugar solution was the white sugar solution. In addition, the sugar solution prepared with dextrose, fructose and sucrose resulted in the failure to produce a colony. The reason for this failure should be investigated through further experimentation.

The present study examines the available sugar solutions for colony development by $B$. terrestris queens. Taken together, the results indicate that the sugar solution with the most favorable effects on the egg-laying and colony developmental characteristics of $B$. terrestris bumblebee queens was the white sugar solution.

\section{References}

Banda HJ, Paxon RJ (1991) Pollination of greenhouse tomatoes by bees. Acta Hortic 288, 194-198.

Bishop JA, Armbruster WS (1999) Themoregulatory abilities of Alaskan bees: effects of size, Phylogeny and ecology. Func Ecol 13, 711-724.

Brian, AD (1952) Division of labour and foraging in Bombus agrorum Fabricius. J. Anim Ecol 21, 223-240.

Buchmann St. L, Hurley JP (1978) A biophysical model for buzz pollination in angiosperms. J Theor Biol 72, 639-657.

Cartar, RV (1991) Colony energy requirements affect response to predation risk in foraging bumblebees. Ethology 87, 90-96

Cartar, RV (1992) Adjustment of foraging and task switching in energy-manipulated wild bumblebee colonies. Anim Behav 44, 75-87.

Dornhaus A, Cameron S (2003) A scientific note on food alert in Bombus transversalis. Apodologie 34, 87-88.

Dornhaus A, Chittka L (2005) Bumble bees (Bombus terrestris) store both food and information in honeypots. Behav Ecol 16, 661-666.

Duchateau MJ, Velthuis HHW (1988) Development and reproductive strategies in Bombus terrestris colonies. Behavior 107, 186-207.

Free JB (1993) Insect pollination of crops. 2nd ed., Academic Press, London.

Goulson (2003) Bumblebees: their behavior and ecology. Oxford University Press, New York.

Griffin RP, Macfarlane RP, Van den Ende HJ (1991) Rearing and domestication of long tongued bumblebee in New Zealand. Acta Horticul 288, 149-153.

Hannan MA, Maeta Y, Hoshikawa K (1998) Feeding behavior and food consumption in Bombus (Bombus) ignitus under artificial condition (Hymenoptera: Apidae). Entomol Sci 1, 27-32.
Harder LD (1983) Functional differences of the proboscides of short- and long-tongued bee (Hymenoptera, Apidea). Can J Zool 61, 580-586.

Heinrich B (1979) Bumblebee economics. Harvard University Press, Cambridge, MA. 245pp

Heinrich B, Heinrich B MJE (1983) Size and caste in temperature regulation by bumblebees. Physiol Zool 56, 552-562.

Iwasaki M (1995) Introduction of commercial bumblebees into Japan. Honeybee Sci. 16, 17-21.

Katayama E (1973) Observations on the brood development in Bombus ignitus (Hymenoptera, Apidae). II. Brood development and feeding habits. Kontyu 41, 203-216.

Katayama E (1975) Egg-laying habits and brood development in Bombus hypocirta (Hymenoptera, Apidae). II. Brood development and feeding habits. Kontyu 43, 478-496.

Michener CD (1974) The social behavior of the bees. Harvard University Press, Cambridge, Mass.

Minitab incorporated company (2000) Minitab user's guide, Minitab Inc., USA.

Ono M, Suzuki N, Sasaki M, Matsuka M (1994) Food processing strategy of bumblebees (Hymenoptera: Apidae). In: Les Insectes Soxiaux (A. Lenoir, G. Amold and M. Lepage, Eds.), Publ. Univ. Paris Nord. pp. 487.

Owen RE (1998) Body size variation and optimal body size of bumblebee queens (Hymenoptera: Apidae). Can J Zool 120, 19-27.

Pereboom JJM, Velthuis HHW, Duchateau MJ (2003) The organization of larval feeding in bumblebees (Hymenoptera, Apidae) and its significance to caste differentiation. Insect Soc 50, 127-133.

Plowright RMS, Pendrel BA(1977) Larval growth in bumble bees (Hymenoptera: Apidae). Can Ent 109, 967-973.

Pomeroy N, Plowright RC (1982) The relation between worker numbers and the production of males queens in the bumble bee Bombus perplexus. Can. J. Zool. 60, 954-957.

Pyke GH (1978) Optimal body size in bumblebees. Oecologica 34, 255-266.

Ribeiro MF, Velthuis HHW, Duchateau MJ Van der Tweel I (1999) Feeding frequency and caste differentiation in Bombus terrestris larvae. Insect Soc. 46, 306-314.

Sutcliffe GH, Plowright RC (1988) The effects of food supply on adult size in the bumblebee Bombus terricola Kirby (Hymenoptera: Apidae). Can Ent 120, 1051-1058.

Sutcliffe GH, Plowright RC (1990) The effects of pollen availability on development time in the bumblebee Bombus terricola Kirby (Hymenoptera: Apidae). Can J Zool. 68, 11201123.

Spaethe J, Weidenmüller A (2002) Size variation and foraging rate in bumblebee (Bombus terrestris). Insect Soc 19, 142146.

Tasei JN (1994) Effect of different narcosis procedures on initiating oviposition of pre-diapausing Bombus terrestris queens. Entomol Exp Appl 72, 273-279.

Tasei J N, Aupinel P (1994) Effect of photoperiodic regimes on the oviposition of artificially overwintered Bombus terrestris 
L. queens and the production of sexuals. J Apicul Res 33, 27-33.

Wheeler DE (1986) Developmenal and physiological determinants of caste in social Hymenoptera: evolutionary implications. Am Nat 128, 13-34.

Whitney HM, Dyer A, Chittka L, Rands SA (2008) The interaction of temperature and sucrose concentration on foraging preference in bumblebees. Naturwissenschaften 95, 845850.

Wide J de, Beetsma J (1982) The physiology of caste development in social insects. J Insect Physiol 16, 167-246.

Wingston ML (1979) The proboscis of the long-tongued bees: a comparative study. Sci Bull Univ Kansas 51, 631-667.

Yoon HJ, Kim SE (2002) Facilitating effects of helpers on oviposition and colony development of bumblebee queen, Bombus ignitus. Koeran J Appl Entomol 41, 239-245.

Yoon HJ, Kim SE, Kim YS (2002) Temperature and humidity favorable for colony development of the indoor-reared bumblebee, Bombus ignitus. Appl Entomol Zool 37, 419-423.
Yoon HJ, Kim SE, Lee SB, Park IG (2003) Effect of $\mathrm{CO}_{2}$-treatment on oviposition and colony development of the bumblebee, Bombus ignitus. Korean J Appl Entomol 42, 139-144.

Yoon HJ, Kim SE, Kim YS, Lee SB (2004a) Colony developmental characteristics of the bumblebee queen Bombus ignitus by the first ovipositon day. Int J Indust Entomol 8, 139143.

Yoon HJ, Lee SB, Kim SE, Seol KL (2004b) The flight of the bumblebee queen, Bombus terrestris, after diapause termination affects to ovipositon and colony development. Int $\mathbf{J}$ Indust Entomol 9, 241-247.

Yoon HJ, Kim SE, Lee SB, Seol KL (2005a) The effect of antiseptic and sugar solutinon on colony development of the bumblebee, Bombus ignitus and B. terrestris. Int J Indust Entomol 11, 43-48.

Yoon HJ, Kim SE, Lee KY, Park IG (2005b) Oviposition and colony development of the bumblebees, Bombus ignitus and $B$. terrestris depending on different pollen. Int J Indust Entomol 11, 99-105. 